\title{
MID-TERM CLINICAL OUTCOMES OF SURGICALLY TREATED MALIGNANT SACRUM TUMORS
}

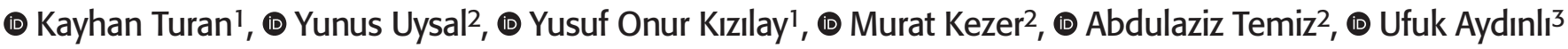 \\ ${ }^{1}$ Istanbul Atlas University Faculty of Medicine, Department of Orthopedics and Traumatology, İstanbul, Turkey \\ 2 Private Hospital Arrhythmia Osmangazi, Clinic of Orthopedics and Traumatology, Bursa, Turkey \\ ${ }^{3}$ Verom Spine and Tumor Center, Bursa, Turkey
}

\begin{abstract}
Objective: There are limited studies in the literature reporting clinical outcomes of malignant sacrum tumors due to their rarity. The aim of this study was to evaluate mid-term clinical outcomes of malignant sacrum lesions surgically treated by a single surgeon.

Materials and Methods: Patients who were surgically treated with the diagnosis of sacrum tumor were retrospectively analyzed. Patients who underwent surgical resection of malignant sacrum tumors were included. Exclusion criteria were incomplete medical records and follow-ups. Patients' demographics, type of the tumor, histopathological diagnosis, presence of neurologic deficit, type of approach, type of resection, presence of lumbopelvic fixation, postoperative complications, and recurrence rates were noted from our medical records.

Results: A total of 15 patients (7 females, 8 males) with a mean age of 43.3 years participated in the study. The most common histopathological diagnosis of the malignant sacrum tumor was chordoma that was seen in 10 of 15 patients (67\%). Soft-tissue tumors (leiomyosarcoma and malignant mesenchymal tumor) were seen in 2 patients (13\%), metastatic tumors (renal cell carcinoma and malignant melanoma) in 2 patients (13\%), and primary osteogenic tumor (chondrosarcoma) in 1 patient (7\%). Perioperative and postoperative complications were encountered in 7 of 15 patients (47\%).

Conclusion: Chordoma was the most encountered malignant tumor of the sacrum according to our findings. Recurrence of the malignant sacrum tumor was so common that half of our patients had a history of previous treatment. It was remarkable that perioperative and postoperative complications were also common.
\end{abstract}

Keywords: Sacrum, malignant, tumor, surgery, spine

\section{INTRODUCTION}

Malignant tumors of the sacrum are a rare clinical entity. Most malignant sacrum tumors are low-grade malignancies, such as chondrosarcomas and chordomas, and a few are high-grade,such as sarcomas and osteosarcomas ${ }^{(1-3)}$. Treatment of these tumors requires a multidisciplinary approach. Oncologists, radiologists, radiotherapists, and spine surgeons must collaborate in diagnosis and treatment. After clinical, morphologic, functional, and laboratory examinations, it is essential to perform a biopsy for diagnosis ${ }^{(4,5)}$. After histological diagnosis, the team decides on personalized treatment.

Treatment protocols may constantly be changing. While the first treatment approach for Ewing's sarcoma was the surgery about ten years ago, it has become the next option after chemotherapy with new chemotherapeutic agents. For chordoma and chondrosarcoma, the surgical approach is still the first choice. While in some cases, en-bloc surgery is sufficient, in others, wide resection is required for long-term survival. The reason for the high number of local recurrences in the complex anatomy of the region, which makes it difficult to surgically reach the entire tumor ${ }^{(1-7)}$.

There are limited studies in the literature reporting clinical outcomes of malignant sacrum tumors due to their rarity. It is important to share information about this rare clinical entity in order to guide orthopedic surgeons. Therefore, the aim of this study was to evaluate mid-term clinical outcomes of malignant sacrum lesions surgically treated by a single surgeon.

\section{MATERIALS AND METHODS}

This retrospective case-series study was performed under the approval of İstanbul Atlas University Ethical Review Board (ID: E-22686390-050.01.04-9540) and conducted in accordance with the Declaration of Helsinki. Informed consent was

Address for Correspondence: Kayhan Turan, İstanbul Atlas University Faculty of Medicine, Department of Orthopedics and Traumatology, İstanbul, Turkey Phone: +90 5393361382 E-mail: kayhan@turanturan.com.tr Received: 22.10.2021 Accepted: 10.11.2021

ORCID ID: orcid.org/0000-0002-1164-6857 
turkishspine

obtained for each patient. Patients who were surgically treated with the diagnosis of sacrum tumors between 2011 and 2019 were retrospectively analyzed. Patients who underwent surgical resection of malignant sacrum tumors were included in the study. Exclusion criteria were incomplete medical records and follow-ups.

Patients' demographics (age, gender), type of the tumor (congenital tumor, primary neurogenic tumor, primary bone tumor, primary soft-tissue tumor, metastatic tumor), histopathological diagnosis, presence of neurologic deficit, type of approach (anterior, posterior, lateral), type of sacrum resection (total, hemicorporectomy, partial, hemisacrectomy), presence of lumbopelvic fixation, postoperative complications and recurrence rates were noted from our medical records.

\section{Statistical Analysis}

Descriptive statistical analysis was performed by using SPSS 25.0 software (SPSS Inc., IBM, NY, USA). Numerical variables were given as means and standard deviations, and categorical variables were given as frequencies and percentages.

\section{RESULTS}

A total of 15 patients (7 females, 8 males) were participated in the current study. The mean age of the patients was $43.3 \pm 16.6$ years old (ranges, 20 to 69 years old). The mean follow-up time was 5.2 \pm 2.9 years (ranges, 3 to 11 years). The most common histopathological diagnosis of the malignant sacrum tumor was chordoma.
Chordoma, was seen in 10 of 15 patients (67\%). Soft-tissue tumors (leiomyosarcoma and malignant mesenchymal tumor) were seen in 2 patients (13\%), metastatic tumors (renal cell carcinoma and malignant melanoma) were seen in 2 patients (13\%), and primary osteogenic tumor (chondrosarcoma) was seen in 1 patient $(7 \%)$.

Eight of 15 patients (53\%) had a history of previous treatment for the diagnosis of malignant sacrum tumor that revealed these patients underwent resection of the recurrent malignant sacrum tumor. The neurogenic deficit was present in 5 of 15 patients (33\%) preoperatively. Operative data of the patients were demonstrated in Table 1.

Perioperative and postoperative complications were encountered in 7 of 15 patients (47\%). The most common perioperative complication was rectum perforation which occurred in 2 of 15 patients. These two patients underwent primary repair by the general surgeon. One patient had a dura mater tear during surgery and it was primarily repaired. The most common of postoperative complication was prolonged drainage and closure defect of the wound that occurred in 3 patients. These patients underwent wound debridement and vacuum-assisted closure treatment. Postoperatively one patient had a neurologic deficit and this patient underwent revision of the screw fixation. During follow-ups, 3 patients had recurrence of the tumor ( 2 chordoma and 1 malignant mesenchymal tumor).

Table 1. Operative data of the patients

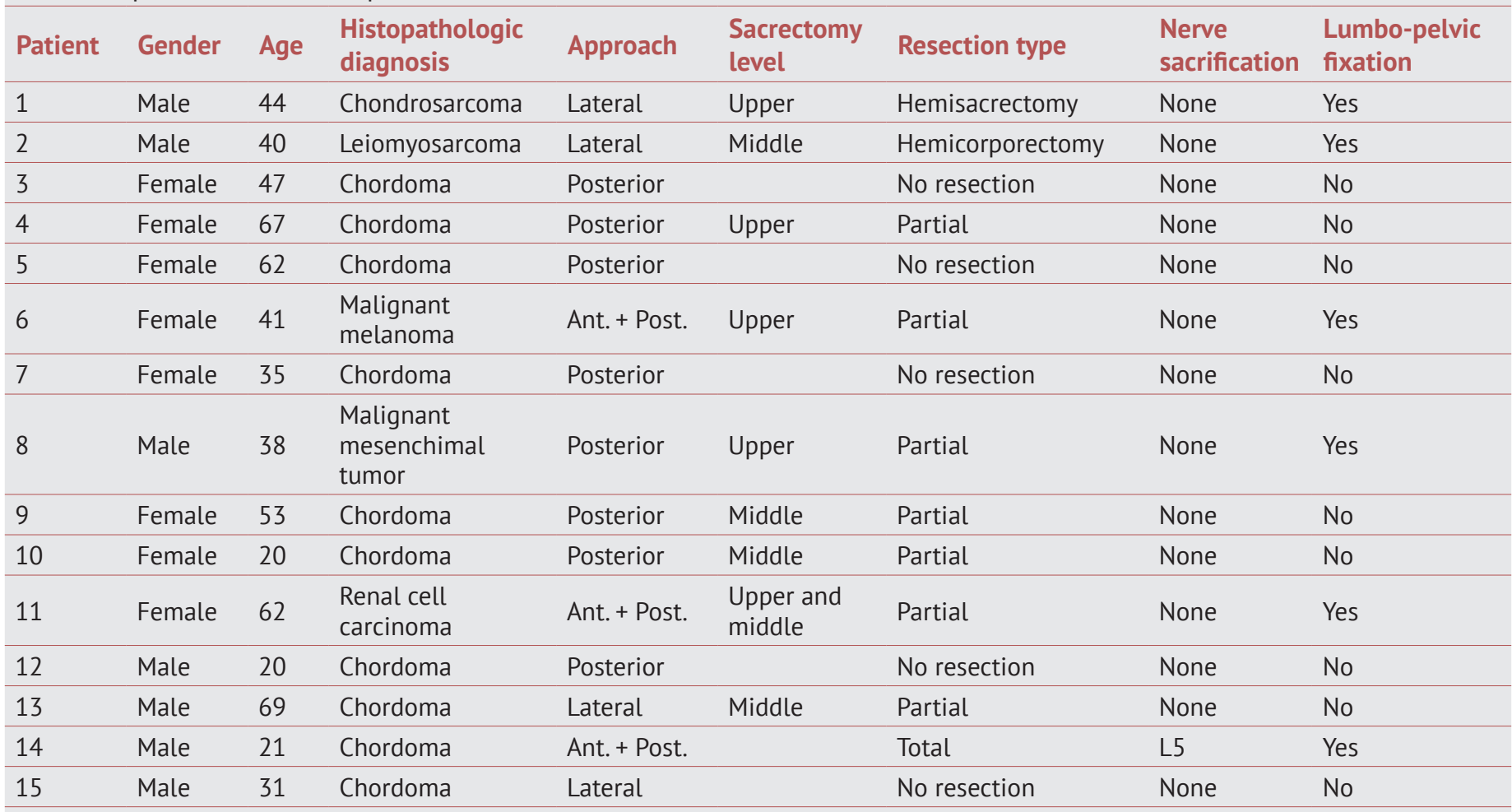

Ant.: Anterior, Post.: Posterior 


\section{DISCUSSION}

Chordomas are the most common histopathological form in sacral tumors. They constitute approximately $40 \%$ of all malignant sacral tumors. Less frequently, sarcomas and other metastatic tumors. In our series, 10 of 15 cases were chordomas (67\%). This tumor originates from the notochord and is aggressive. Although it is considered a tumor, grows slowly(5). The most prominent symptom is hip and leg pain. Pain occurs when the tumoral tissue compresses the surrounding tissues. Neurological symptoms are independent of pain ${ }^{(7,8)}$.

Combined techniques are generally used in the treatment. The tumor size, localization, borders, and character of the tumor play a role in the selection of treatment. Chordomas and chondrosarcomas are tumors that are resistant to radiotherapy and chemotherapy, but radiotherapy and chemotherapy are applied as adjuvant treatment after resection surgery. Especially after partial and subtotal resections, the success of the treatment, and longer survival without long-term recurrence, adjuvant therapy is necessary ${ }^{(1,7,8)}$. In our series, all our patients received adjuvant radiotherapy and chemotherapy. New chemotherapeutic agents are effective both in increasing the effectiveness of surgery and in obtaining more successful results in cases where surgery cannot be performed $(3,9,10)$.

Surgical approaches are classically anterior, posterior, lateral, and combined approaches. Combined approaches are preferred for more successful results and long-term survival. An anterior approach is required if the tumor has invaded the pelvic organs. If spinopelvic fixation is required, posterior and combined approaches are recommended. In our series, a posterior approach was required in 8 cases, a lateral approach in 4 cases, and a combined approach in 3 cases. Depending on the location of the tumor, sacrectomy levels vary, and nerve root sacrification may sometimes be inevitable in more proximal tumors. Stabilization may be necessary in cases where lumbosacral instability develops ${ }^{(11,12)}$. Lumbosacral fixation also provides an advantage in terms of postoperative quality of life for the patient ${ }^{(12)}$. We performed lumbosacral stabilization in a case where the tumor borders exceeded the lumbosacral junction and we had to sacrifice the L5 nerve root. If more than $50 \%$ of the sacroiliac joint is removed and lumbosacral instability occurs, stabilization is required ${ }^{(11-14)}$.

In cases where the resection is large, a rectus abdominis myocutaneous flap is used to close the defect area. We used the rectus flap for defect closure in two of our cases where we performed high sacrectomy with the anterior-posterior combined approach, and we achieved successful results. Early postoperative surgical site infection is one of the most common complications. Being close to the perineum facilitates infection. Wide resection and cavity formation also pave the way for infection. The early infection rate in the literature is between $25 \%$ and $50 \%$. In our series, early infection was found to be $36 \%$, similar to the literature. The infection rate in intralesional resections is significantly lower. In addition, lumbopelvic fixation can prevent instability and reduce infection formation ${ }^{(15-17)}$.

For postoperative rehabilitation and ambulation, the preservation of the L5-S1 nerve root and the preservation of the S2 nerve root are necessary and important for sphincter function. While the preservation of the nerve roots is necessary for the functions, on the other hand, sacrificing the nerve root and performing wider resection is important in preventing local recurrences ${ }^{(16,17)}$. In our series, the $L 5$ nerve root was sacrificed in only 1 case. Our view is to preserve the $L 5$ nerve root as much as possible for functional results. We tried to resection from the widest possible border while trying to preserve the nerve root as much as possible.

\section{Study Limitations}

The main limitations of our study were its the limited number of cases and retrospective design. However, we reported outcomes of a rare clinical entity, the surgical treatment of malignant sacrum tumor, in which outcomes of treatments as well as giving information about potential complications are very important for spine surgeons. Further studies with larger cohorts are needed in order to better assess outcomes, complications, and recurrence rates.

\section{CONCLUSION}

Chordoma is the most encountered malignant tumor of the sacrum according to our findings. Recurrence of the malignant sacrum tumor was so common that half of our patients had a history of previous treatment. It is remarkable that perioperative and postoperative complications were also common in our series, even if surgical treatment had been performed by an experienced spine surgeon.

\section{Ethics}

Ethics Committee Approval: Ethics committee approval was obtained from İstanbul Atlas University Ethical Review Board (ID: E-22686390-050.01.04-9540).

Informed Consent: Informed consent was obtained for each patient.

Peer-review: Internally peer-reviewed.

\section{Authorship Contributions}

Surgical and Medical Practices: U.A., Concept: K.T., U.A., Design: K.T., U.A., Data Collection or Processing: Y.U., Y.O.K., M.K., Analysis or Interpretation: K.T., Y.U., Y.O.K., M.K., A.T., Literature Search: A.T., Writing: K.T.

Financial Disclosure: The authors declared that this study received no financial support.

Conflict of Interest: The authors have no conflicts of interest to declare.

\section{REFERENCES}

1. Varga PP, Szövérfi Z, Lazary A. Surgical treatment of primary malignant tumors of the sacrum. Neurol Res. 2014;36:577-87. 
turkishspine

2. Varga PP, Bors I, Lazary A. Sacral tumors and management. Orthop Clin North Am. 2009;40:105-23.

3. Sciubba DM, Petteys RJ, Garces-Ambrossi GL, Noggle JC, McGirt MJ, Wolinsky JP, et al. Diagnosis and management of sacral tumors. J Neurosurg Spine. 2009;10:244-56.

4. Ruggieri P, Angelini A, Ussia G, Montalti M, Mercuri M. Surgical margins and local control in resection of sacral chordomas. Clin Orthop Relat Res. 2010;468:2939-47.

5. Thornton E, Krajewski KM, O'Regan KN, Giardino AA, Jagannathan JP, Ramaiya N. Imaging features of primary and secondary malignant tumours of the sacrum. Br J Radiol. 2012;85:279-86.

6. Damron TA, Ward WG, Stewart A. Osteosarcoma, chondrosarcoma, and Ewing's sarcoma: National Cancer Data Base Report. Clin Orthop Relat Res. 2007:459:40-7.

7. Ilaslan $H$, Sundaram M, Unni KK, Dekutoski MB. Primary Ewing's sarcoma of the vertebral column. Skeletal Radiol. 2004;33:506-13.

8. Combs SE, Kalbe A, Nikoghosyan A, Ackermann B, Jäkel O, Haberer $\mathrm{T}$, et al. Carbon ion radiotherapy performed as re-irradiation using active beam delivery in patients with tumors of the brain, skull base and sacral region. Radiother Oncol. 2011;98:63-7.

9. Stacchiotti S, Casali PG. Systemic therapy options for unresectable and metastatic chordomas. Curr Oncol Rep. 2011;13:323-30.

10. Stacchiotti S, Longhi A, Ferraresi V, Grignani G, Comandone A, Stupp $\mathrm{R}$, et al. Phase II study of imatinib in advanced chordoma. J Clin Oncol. 2012;30:914-20.
11. Clarke MJ, Dasenbrock H, Bydon A, Sciubba DM, McGirt MJ, Hsieh PC, et al. Posterior-only approach for en bloc sacrectomy: clinical outcomes in 36 consecutive patients. Neurosurgery. 2012;71:35764; discussion 364.

12. Court C, Bosca L, Le Cesne A, Nordin JY, Missenard G. Surgical excision of bone sarcomas involving the sacroiliac joint. Clin Orthop Relat Res. 2006;451:189-94.

13. Guo Y, Palmer JL, Shen L, Kaur G, Willey J, Zhang T, et al. Bowel and bladder continence, wound healing, and functional outcomes in patients who underwent sacrectomy. I Neurosurg Spine. 2005;3:106-10.

14. Guo W, Tang X, Zang J, Ji T. One-stage total en bloc sacrectomy: a novel technique and report of 9 cases. Spine (Phila Pa 1976). 2013;38:E626-31. doi: 10.1097/BRS.0b013e31828b7045.

15. Sar C, Eralp L. Surgical treatment of primary tumors of the sacrum. Arch Orthop Trauma Surg. 2002;122:148-55.

16. Wang J, Tang Q, Xie X, Yin J, Zhao Z, Li Z, et al. Iliosacral resections of pelvic malignant tumors and reconstruction with nonvascular bilateral fibular autografts. Ann Surg Oncol. 2012;19:4043-51.

17. Li D, Guo W, Qu H, Yang R, Tang X, Yan T, et al. Experience with wound complications after surgery for sacral tumors. Eur Spine J. 2013;22:2069-76. 